\title{
Serum lipid profile and insulin resistance in women with polycystic ovary syndrome (PCOS)
}

\begin{abstract}
Background and purpose: Polycystic ovary syndrome (PCOS), a common endocrine disorder with lipid disturbances, is associated with the increased risk of cardiovascular disease and central obesity. The aim of this study was to determine lipid profile, fasting plasma glucose, and insulin resistance in women with PCOS in comparison with a control group.
\end{abstract}

Materials and methods: This case-control study was conducted on 153 women with PCOS and 449 healthy women as controls. Data was extracted from data center of Diabetes Research Center of Ahwaz University of Medical Sciences including women from 4 cities of Khuzestan province (Ahwaz, Behbahan, Abadan, and Khorramshahr). Serum lipids, plasma fasting glucose, and serum insulin levels along with Body Mass Index (BMI), Homeostatic Model Assessment Insulin Resistance (HOMA IR), Lipid Accumulation Product (LAP), and Body Adiposity Index were compared using appropriate statistical tests.

Results: The frequency of low HDL and high total cholesterol was higher in women with PCOS than control group ( $\mathrm{p}=0.032$, and $\mathrm{p}=0.001$, respectively). No statistically significant difference was found between two studied groups in the mean levels of total cholesterol, triglyceride, HDL cholesterol, LDL cholesterol, fasting glucose, HOMA IR, LAP, and PA ( $p>0.05)$. In women with $\mathrm{BMI}<25$ and BMI between 25 and 30 , the mean level of total cholesterol was higher in PCOS group than control group, whereas there was no significant difference in women with BMI $>30$. In women with BMI $>30$, the mean triglyceride and glucose levels was higher in PCOS group than control group $(\mathrm{p}=0.029$, and $\mathrm{p}=0.010)$.

Conclusion: In obese women, the triglyceride and fasting glucose levels are higher in patients with polycystic ovary syndrome than healthy women. In non-obese women, however, the total cholesterol level is higher in patients with polycystic ovary syndrome than healthy women.

Keywords: polycystic ovary syndrome, lipid profile, fasting plasma glucose, insulin resistance, obesity, metabolic syndrome
Volume 5 Issue 3 - 2018

\author{
Homeira Rashidi,' Mina Tafazoli, ${ }^{2}$ Mohammad \\ Taha Jalali, ${ }^{3}$ Amir Mohammad Eghbalnejad \\ Mofrad ${ }^{4,5}$ \\ 'Endocrinologist, Ahvaz Jundishapur University of Medical \\ Sciences, Ahvaz, Iran \\ ${ }^{2}$ Internal Medicine Specialist, Ahvaz Jundishapur University of \\ Medical Sciences, Iran \\ ${ }^{3}$ Professor of Clinical Biochemistry, Ahvaz Jundishapur University \\ of Medical Sciences, Iran \\ ${ }^{4}$ Student Research Committee, Ahvaz Jundishapur University of \\ Medical Sciences, Iran \\ ${ }^{5}$ Medicine department, Ahvaz Jundishapur University of Medical \\ Sciences, Iran
}

Correspondence: Homeira Rashidi, Endocrinologist, Assistant Professor, Health Research Institute, Diabetes Research Center, Ahvaz Jundishapur University of Medical Sciences, Ahvaz, Iran, Post Code: 6I357-15794, Fax 06I-33369539, Tel 09I23 I 42337 Email hrashidi2002@gmail.com

Received: March 05, 2017 | Published: June 28, 2018

\section{Introduction}

For the first time in 1935, the classic form of polycystic ovary syndrome (PCOS) was described by Stein and Levental. They reported the association between polycystic ovaries and amenorrhea, hirsutism and obesity. ${ }^{1}$ Polycystic ovary syndrome encompasses a spectrum of diseases that are associated with the following features: cutaneous hyperandrogenism (such as hirsutism, acne resistant to treatment, or male or female pattern baldness (androgenetic baldness), irregular menstruation (such as oligomenorrhea or amenorrhea, or irregular bleeding), polycystic ovaries (one or two), obesity and insulin resistance. ${ }^{2}$ Polycystic ovary syndrome is the most common endocrine disorder in women with a prevalence of $6-10 \%$ of women in reproductive age and also is the main leading cause of infertility due to lack of ovulation. ${ }^{3,4}$ There is evidence that PCOS is may be the result of metabolic disorders including insulin resistance. Hyperinsulinemia and early incidence of type 2 diabetes in patients with this syndrome is higher than the normal population. ${ }^{2}$ Given the role of insulin resistance in polycystic ovary, lowering medications are used to treat insulin resistance..$^{5-7}$ Dyslipidemia pattern in insulin resistance, low levels of HDL and high triglyceride levels pattern are common in women with polycystic ovary syndrome. Hyperinsulinemia inhibits lipolysis and thus increase esterified acids. High levels of nonesterified fatty acids (NEFA) may increase triglyceride and decrease HDL level. ${ }^{8}$ Dyslipidemia is a common metabolic disorder in women with polycystic ovary syndrome, although in different geographic regions and ethnic groups has been reported different. ${ }^{9}$ Abnormal situation of lipoproteins is common in this syndrome, these disorders include: Increased level of total cholesterol, triglycerides, LDL (lowdensity lipoprotein), reduced high-density lipoprotein and apoprotein A1. ${ }^{9}$ Abdominal obesity is positively associated with increased LDL and VLDL and has inverse proportion with amount of HDL. ${ }^{10}$

About half of women are suffering from obesity syndrome. ${ }^{3} \mathrm{~A}$ large number of adolescents with PCOS have overweight or obese. In fact, the most common endocrine causes of obesity in adolescent is PCOS..$^{11}$ Obesity has a major impact on PCOS patient phenotype and possibly have dominant role in physiopathology of hyperandrogenism, chronic anovulation and metabolic system. Obesity clearly associated with increased infertility, metabolic syndrome and increased risk of cardiovascular disease. ${ }^{12,13}$ A study showed that women with polycystic ovary syndrome and obese had higher risk for developing hyperandrogenism, insulin resistance, hypercholesterolemia, hypertriglyceridemia and high reactive protein $\mathrm{C}(\mathrm{CRP})$ compared to 
people with normal weight. ${ }^{14}$ Also results of a meta-analysis showed that the metabolic and reproductive outcomes in women with PCOS and obese were worsen compared to group with normal weight. ${ }^{15}$ Lose weight and increased physical exercises have been caused improving hyperandrogenism, insulin resistance and ovarian function in patients with PCOS..$^{15,16}$ In this study, the prevalence and association of dyslipidemia, insulin resistance and related indices were compared in 18-45 year-old women with and without polycystic ovary syndrome of Khuzestan province in southern of Iran considering the geographic and racial differences of these cases.

\section{Method}

In this case-control study, existing data on the research project No. D-88025 from Diabetes Research Center of Ahvaz University of Medical Sciences was used. Intended study was investigated and verified at the university ethics committee. Data were belonging to 602 women 18-45 years old (in childbearing age) of 4 city of the province (400 persons from Ahvaz, 100 persons from Behbahan and 100 persons from Abadan and Khorramshahr). Among these 153 patients were with polycystic ovary syndrome (case) and 449 persons were healthy (control) that the control group was matched in terms of age with case group. According to Rotterdam criteria, existence of two features of oligioovulation characteristics (cycle length is more than 35 days), clinical or laboratory hyperandrogenism (clinical symptoms of hirsutism, acne and male pattern hair loss or increased level of testosterone and or dehydroepiandrosterone) and a morphological view of PCOS on ovaries sonography, PCOS diagnosis was confirmed. Hirsutism, the growth of terminal hair in androgendependent areas, behind the upper lip, chin, trunk, abdomen, arms and thighs that both patients and physicians were considered them abnormal were included and according to Ferriman-Gallway Score was defined and graded. Hirsutism was applied to F-G score greater or equal to 7 . Transvaginal sonography was performed during the first 5 days of the normal menstrual cycle or created menstrual after taking oral or injectable progesterone for a week. Sonography result according to the Rotterdam criteria, if it exists 12 or more follicles in each ovary with a diameter of 2 to $9 \mathrm{~mm}$ or ovarian volume was greater than $10 \mathrm{~mm}$ was considered as polycystic ovaries. Catching other causes of hyperandrogenism, such as congenital adrenal hyperplasia, Cushing's syndrome, ovarian or adrenal tumor, taking medications that leading to symptoms as menstrual disorders, hirsutism or acne, pregnancy, menopause and use of medications that affect the lipid profile or blood sugar during the past three months (like OCP, thiazides, corticosteroids, metformin and so on) leading to withdrawal of individuals from the study.

Assessed variables including age, anthropometric measurements such as height, weight, waist and hip circumference, serum lipids, fasting plasma glucose and serum insulin level. Body mass index (BMI) between 18 to 25 were normal, BMI values over 30 were considered as obese and BMI between 25 and 30 were considered as overweight. The fasting plasma glucose, triglycerides, cholesterol, LDL and HDL were done after 12 to 14 hours of fasting. dyslipidemia by definition NCEP, triglycerides greater than or equal to $200 \mathrm{mg} / \mathrm{dL}$, HDL cholesterol lower than $35 \mathrm{mg} / \mathrm{dL}$, LDL cholesterol higher than $130 \mathrm{mg} / \mathrm{dL}$ or total cholesterol more than $200 \mathrm{mg} / \mathrm{dL}$ were considered as disorder. Diabetes as FBS was defined more than or equal to $126 \mathrm{mg} / \mathrm{dL}$. Using existing data, BMI, HOMA IR, LAP index and BA index indicators were calculated by the formula. Finally, all data were collected and analyzed using statistical tests.

Data were entered by an operator in statistical software SPSS (version 19). Then, by using descriptive statistics (mean, standard deviation, and frequency) data was summarize and describe. A normal distribution of test values was investigated using One-Sample Kolmogorov-Smirinov. Independent t test was used to compare the mean differences of quantitative variables between two groups and the chi-square test and Fischer's exact test was used to evaluate the association between qualitative variables of study with polycystic ovary syndrome catching. 0.05 was considered significant.

\section{Results}

Of 602 women with a mean age of 33 years were 153 polycystic ovary syndrome (cases) and 449 healthy people (control group). Of602subjects, 28 people of normal weight (BMI $<25$ ), 296 overweight people $(30>$ BMI $>25)$ and 278 were obese (BMI> 30), respectively. The mean age of patients with polycystic ovary syndrome and non PCOs were $32 \pm 7.8 \mathrm{SD}$ and $34 \pm 7.3 \mathrm{SD}$ years, between the two groups in terms of age, there was no statistically significant difference In Table 1, the mean level of serum lipids, glucose and HOMA index in terms of polycystic ovary syndrome catching in groups with body mass index less than 25, between 25 to 30 and above 30 and also in all persons along with $\mathrm{p}$ values that obtained from independent $\mathrm{t}$ test have been shown. Average LDL level in patients with polycystic ovary syndrome were $30.51 \pm 101.80 \mathrm{mg} / \mathrm{dL}$ and in healthy controls were $30.01 \pm 97.03 \mathrm{mg} / \mathrm{dL}$. Independent t tests were not shown statistically significant differences between two groups $(\mathrm{p}=0.096)$.

Table I Comparison of mean of lipid, glucose and IR HOMA index in BMI between patients with PCOs and nonPCOs

\begin{tabular}{|c|c|c|c|c|c|c|c|c|c|}
\hline & & $\mathrm{BMI}<25^{\prime}$ & & $25=<\mathrm{BMI}<$ & & BMI > $=30^{3}$ & & TOTAL & \\
\hline & & Mean (SD) & (p value) & Mean (SD) & ( $p$ value) & Mean (SD) & (p value) & Mean (SD) & (p value) \\
\hline \multirow{4}{*}{ T-Cholesterol } & & 162.6 & \multirow{4}{*}{0.026} & 158.32 & \multirow{4}{*}{0.047} & 170.53 & \multirow{4}{*}{0.15} & 167.44 & \multirow{4}{*}{0.182} \\
\hline & PCOs & (27.23) & & (33.68) & & (34.93) & & (35.19) & \\
\hline & \multirow{2}{*}{ nonPCOs } & 197.16 & & 168.75 & & 171.11 & & 171.81 & \\
\hline & & $(35.40)$ & & $(36.15)$ & & $(30.47)$ & & (32.95) & \\
\hline \multirow{4}{*}{ TG } & & 94.86 & \multirow{4}{*}{0.266} & 95.66 & \multirow{4}{*}{0.331} & $123.5 \mid$ & \multirow{4}{*}{0.029} & 117.53 & \multirow{4}{*}{0.325} \\
\hline & PCOs & (43.92) & & (56.08) & & $(63.16)$ & & (8I.II) & \\
\hline & \multirow{2}{*}{ nonPCOs } & 122 & & 103.94 & & 131.25 & & 124.67 & \\
\hline & & $(60.91)$ & & $(55.14)$ & & $(53.39)$ & & $(63.52)$ & \\
\hline
\end{tabular}


Table continuded

\begin{tabular}{|c|c|c|c|c|c|c|c|c|c|}
\hline & & $B M I<25^{\prime}$ & & $25=<\mathrm{BMI}<30^{2}$ & & BMI $>=30^{3}$ & & TOTAL & \\
\hline \multirow{5}{*}{ HDL } & & 51.86 & \multirow{5}{*}{0.337} & 50.17 & \multirow{5}{*}{0.393} & 45.5 & \multirow{5}{*}{0.392} & 47.17 & \multirow{5}{*}{0.143} \\
\hline & PCOs & (12.67) & & $(12.10)$ & & (II.26) & & (11.96) & \\
\hline & & & & & & & & & \\
\hline & nonPCOs & 46 & & 48.62 & & 44.55 & & 45.53 & \\
\hline & & $(11.26)$ & & $(11.27)$ & & (II.87) & & (II.47) & \\
\hline \multirow{7}{*}{ FBS } & & 102.4 & \multirow{7}{*}{0.411} & 95.08 & \multirow{7}{*}{0.394} & 98.82 & \multirow{7}{*}{0.01} & 99.38 & \multirow{7}{*}{0.794} \\
\hline & PCOs & & & & & & & & \\
\hline & & (20.28) & & (41.58) & & (37.56) & & $(49.96)$ & \\
\hline & & & & & & & & & \\
\hline & \multirow{3}{*}{ nonPCOs } & 95 & & 90.34 & & 100.21 & & 98.27 & \\
\hline & & & & & & & & & \\
\hline & & $(10.58)$ & & $(12.02)$ & & $(24.3 \mathrm{I})$ & & (26.64) & \\
\hline \multirow{7}{*}{ HOMA-IR } & & 4.36 & \multirow{7}{*}{0.367} & 1.8 & \multirow{7}{*}{0.494} & 2.25 & \multirow{7}{*}{0.198} & 2.34 & \multirow{7}{*}{0.2} \\
\hline & $\mathrm{PCOs}$ & & & & & & & & \\
\hline & & (6.340) & & (2.25) & & (2.11) & & (2.82) & \\
\hline & \multirow{4}{*}{ nonPCOs } & & & & & & & & \\
\hline & & 1.89 & & 2.04 & & 2.94 & & 2.7 & \\
\hline & & & & & & & & & \\
\hline & & (1.68) & & (2.06) & & (3.57) & & (3.01) & \\
\hline
\end{tabular}

HDL, high density lipoprotein; HOMA IR, homeostatic model assessment insulin resistance

Table 2 The mean of Serum Insulin, LAP and I BA in polycystic ovary syndrome

\begin{tabular}{llllll}
\hline & Condition & Number & Average & Standard deviation & Different (p value) \\
\hline \multirow{2}{*}{ LAP } & Control & 424 & 2995.78 & 3257.01 & 0.185 \\
& Case & 153 & 3376.19 & 2742.13 & 0.204 \\
BAI & Control & 424 & 181.97 & 27.23 & 0.171 \\
& Case & 153 & 185.63 & 26.82 & 9.27 \\
\multirow{2}{*}{ Serum Insulin } & Control & 424 & 9.41 & 10.31 & \\
& Case & 153 & 10.64 & & \\
\hline
\end{tabular}

LAP, lipid accumulation product index; BAI, body adiposity index

In Table 2, Average serum insulin level (Serum Insulin), index of lipid accumulation product (LAP) and the index of adiposity (BA) has been presented. As can be seen, an independent $t$ test, showed no statistically significant differences between the two case and control groups in terms of these indicators ( $p$ values $>0.05$ ). Figure 1 shows the frequency of serum lipid disorders among women with PCOS and healthy women. There is a statistically significant association between polycystic ovary syndrome and the status of high total cholesterol and low HDL ( $p$ values are 0.001 and 0.032 , respectively), but there is no statistically correlation between polycystic ovary syndrome and triglycerides state $(\mathrm{p}=0.225)$.

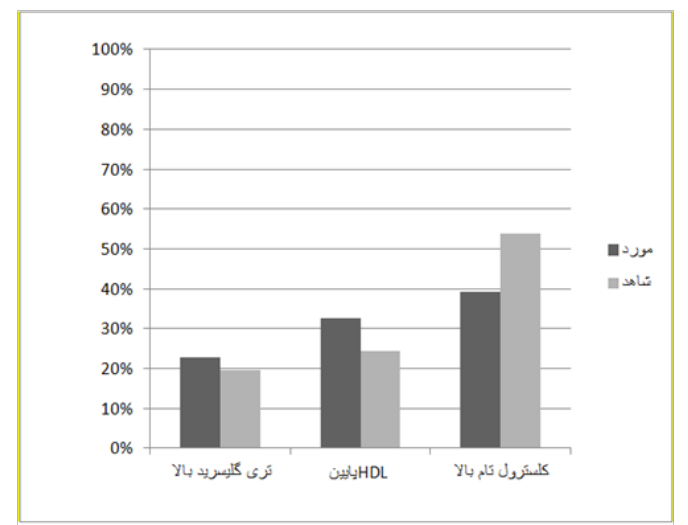

Figure I The prevalence of peruvial imbalances in the treatment of individuals with a reading resource.

\section{Discussion}

In this study, $34.6 \%$ of PCOS patients were obese. In our study, body mass index between two groups of patients with polycystic ovary syndrome and control group had no significant difference. In our study there was a statistically significant association between high serum total cholesterol and low HDL with polycystic ovary syndrome, but this association was not true in the case of triglycerides and high LDL. Also mean of serum lipid levels in case and control groups were not significantly different. Of course, when the results were separated based on body mass index, significant differences were observed in average of serum triglycerides in BMI $>30$, and also in total serum cholesterol in patients with $\mathrm{BMI}<25$ and $25<\mathrm{BMI}<30$; in all these subjects, case group had higher levels than the control group. Several studies have been evaluated dyslipidemia in women with polycystic ovary syndrome. Although it seems that different studies have reported conflicting results due to factors such as race, genetics, nutrition, lifestyle and economic differences in their study population.

Results of studies by Dunaif et al., ${ }^{17}$ Roa et al. ${ }^{18}$ in 2009 in Venezuela, Talbott et al., ${ }^{19}$ Orio et al. ${ }^{20}$ in Italy, Legro et al., ${ }^{21}$ Erel et al. ${ }^{22}$ in Turkey, are generally consistent with the results of our study that in all, the levels of serum lipids (total cholesterol, LDL, triglycerides) in patients with polycystic ovary were higher and level of HDL was lower than healthy persons. However, in our study, this difference was not statistically significant in some cases. In study of lankarani et al., ${ }^{23}$ that was conducted in 2004 , in contrast to our study, mean body mass index and triglyceride level have significant different 
between two groups of women with PCOS and healthy women. But as our study, there is no statistically significant difference between fasting serum glucose, total cholesterol, LDL and HDL in both groups. In this study, after removing the effect of BMI, no significant differences were observed between the triglyceride level in case and control groups. In the study by Javadian et al. ${ }^{24}$ in 2011, women with polycystic ovary had higher levels of postprandial triglycerides, postprandial cholesterol, fasting blood glucose, insulin and waist-tohip ratio compared with the healthy control group. In an overnight fasting, triglycerides and cholesterol were also higher in patients than the control group. There was no significant correlation between the measured lipid level and insulin level and waist-to-hip ratio.

In the study of Rocha et al. ${ }^{25}$ In 2011, the incidence of dyslipidemia in patients with this syndrome was twice more than control group (76.1\% vs. $32.25 \%)$, that mostly as a decrease in HDL (57.6\%) and increases in triglycerides $(28.3 \%$ ) but there is no significant differences in total cholesterol and LDL and triglyceride level in sub-groups. BMI had a significant effect on these disorders. In our study, the prevalence of high triglyceride and low HDL in case group was more than control (insignificantly) but the prevalence of high serum total cholesterol was significantly higher in the control group.

In this study, the prevalence of impaired fasting glucose in patients with PCOS was $3 \%$ and $2 \%$ in the control group that had no statistically significant difference. Although mean fasting blood glucose and mean of serum insulin level were higher in the case group, but this difference was not statistically significant. In a study conducted by the doctor Bahrami et al., ${ }^{26}$ the prevalence of diabetes in women with PCOS was $4.3 \%$, which was significantly higher than the control group. In the Morrin-Papunen and colleagues study ${ }^{27}$ in 2000 , the prevalence of impaired fasting glucose (IFG) there was no significant difference between obese and thin women with PCOS and thin and obese persons of control group. In the study of Velazquez and colleagues, ${ }^{28}$ both obese and non-obese women with polycystic ovary syndrome, had higher insulin levels than the control group. In Nazari et al. ${ }^{29}$ study on 60 women with PCOS and 60 healthy women, insulin level and fasting blood glucose was significantly higher in case group. Studies show that measurement of plasma fasting glucose is not a sensitive screening test for diagnosis of diabetes in patients with PCOS. ${ }^{30}$ Also in our study, a significant association between fasting blood sugar and polycystic ovary syndrome was not found. It is possible that with standard oral glucose tolerance test (OGCT) in these patients showed the glucose tolerance disorders better. The important reason for difference between our results with other research is that this study was population base, but other studies were clinical base. According to the presented results it is suggested that the risk of emergence of glucose metabolism disorders be reminded to all patients with PCOS and the need for lifestyle modification be recommended and is also recommended to all women with PCOS, regardless of age and body mass index screened in terms of metabolic disorders including diabetes and hyperlipidemia.

\section{Conclusion}

Triglyceride and glucose levels in obese women and total cholesterol level in non-obese women with polycystic ovarian syndrome were more than healthy women.

\section{Acknowledgments}

This article is taken from Mrs. Dr. Mina Tafazoli thesis (registration number: 1060/D). This thesis has been approved by university of Ahwaz Jondishapour Medical Sciences. Authors appreciate for financial support from department of Development, Research and Technology, university of Jondishapour Medical Sciences.

\section{Conflict of interest}

Author declares there is no conflict of interest.

\section{References}

1. Melmed S, Polonsky KS, Larsen PR. Williams's textbook of endocrinology. Elsevier Health Sciences; 2011.

2. Ovalle F, Azziz R. Insulin resistance, polycystic ovary syndrome, and type 2 diabetes mellitus. Fertil Steril. 2002;77(6):1095-1105.

3. Baptiste CG, Battista MC, Trottier A, et al. Insulin and hyperandrogenism in women with polycystic ovary syndrome. J Steroid Biochem Mol Biol. 2010;122(1):42-52.

4. Scarpitta AM, Sinagra D. Polycystic ovary syndrome: an endocrine and metabolic disease. Gynecol Endocrinol. 2000;14(5):392-395.

5. Nazari T, Bayat R, Hamedi M. Metformin therapy in girls with polycystic ovary syndrome: a self-controlled clinical trial. Arch Iran Med. 2007;10(2):176-181.

6. Amato MC, Galluzzo A, Merlino S, et al. Lower insulin sensitivity differentiates hirsute from non-hirsute Sicilian women with polycystic ovary syndrome. Eur J Endocrinol. 2006;155(6):859-865.

7. Gambineri A, Pelusi C, Genghini S, et al. Effect of flutamide and metformin administered alone or in combination in dieting obese women with polycystic ovary syndrome. Clin Endocrinol (Oxf). 2004;60(2):241249

8. De Leo V, la Marca A, Petraglia F. Insulin-lowering agents in the management of polycystic ovary syndrome. Endocr Rev. 2003;24(5):633667.

9. Sam S, Legro RS, Bentley-Lewis R, et al. Dyslipidemia and metabolic syndrome in the sisters of women with polycystic ovary syndrome. J Clin Endocrinol Metab. 2005;90(8):4797-4802.

10. Ibáñez L, de Zegher F. Ethinylestradiol-drospirenone, flutamidemetformin, or both for adolescents and women with hyperinsulinemic hyperandrogenism: opposite effects on adipocytokines and body adiposity. J Clin Endocrinol Metab. 2004;89(4):1592-1597.

11. McCartney CR, Prendergast KA, Chhabra S, et al. The association of obesity and hyperandrogenemia during the pubertal transition in girls: obesity as a potential factor in the genesis of postpubertal hyperandrogenism. J Clin Endocrinol Metab. 2006;91(5):1714-1722.

12. Barber TM, McCarthy MI, Wass JAH, Franks S. Obesity and polycystic ovary syndrome. Clin Endocrinol (Oxf). 2006;65(2):137-145.

13. Costa EC, de Sá JC, Mafaldo Soares EM, et al. Anthropometric indices of central obesity how discriminators of metabolic syndrome in Brazilian women with polycystic ovary syndrome. Gynecol Endocrinol. 2012;28(1):12-15.

14. Pavicic Baldani D, Skrgatic L, Sprem Goldstajn M, et al. Clinical and biochemical characteristics of polycystic ovary syndrome in Croatian population. Coll Antropol. 2012;36(4):1413-1418. 
15. Lim SS, Norman RJ, Davies MJ, et al. The effect of obesity on polycystic ovary syndrome: a systematic review and meta-analysis. Obes Rev. 2013;14(2):95-109.

16. Moran LJ, Harrison CL, Hutchison SK, et al. Exercise decreases antimullerian hormone in anovulatory overweight women with polycystic ovary syndrome: a pilot study. Horm Metab Res. 2011;43(13):977-979.

17. Dunaif A, Segal KR, Futterweit W, et al. Profound peripheral insulin resistance, independent of obesity, in polycystic ovary syndrome. Diabetes. 1989;38(9):1165-1174.

18. Roa BM, Arata-Bellabarba G, Valeri L, et al. [Relationship between the triglyceride/high-density lipoprotein-cholesterol ratio, insulin resistance index and cardiometabolic risk factors in women with polycystic ovary syndrome]. Endocrinol Nutr. 2009;56(2):59-65.

19. Talbott E, Guzick D, Clerici A, et al. Coronary heart disease risk factors in women with polycystic ovary syndrome. Arterioscler Thromb Vasc Biol. 1995;15(7):821-826.

20. Orio F, Palomba S, Spinelli L, et al. The cardiovascular risk of young women with polycystic ovary syndrome: an observational, analytical, prospective case-control study. J Clin Endocrinol Metab. 2004;89(8):3696-3701.

21. Legro RS, Kunselman AR, Dunaif A. Prevalence and predictors of dyslipidemia in women with polycystic ovary syndrome. Am J Med. 2001;111(8):607-613

22. Erel CT, Senturk LM, Kaleli S, et al. Is serum leptin level regulated by thyroid functions, lipid metabolism and insulin resistance in polycystic ovary syndrome? Gynecol Endocrinol. 2003;17(3):223-229.
23. Muhannaz L, Nada Waz, Ramin H. Determination of diplidemi dersenderme. Diabet Magazine and Metabolism of Iran. Diabet and Lepid Iran Magazine.

24. M Javadian, Z Basirat, M Kashifard. Relationship between the Postprandia Lipid Level with Waist-to-Hip Ratio and Insulin in Patients with Polycystic Ovary Syndrome. J Maz Univ Med Sci. 2011;21(85):55-60.

25. Rocha MP, Marcondes JAM, Barcellos CRG, et al. Dyslipidemia in women with polycystic ovary syndrome: incidence, pattern and predictors. Gynecol Endocrinol. 2011;27(10):814-819.

26. Bahrami A. Prevalence of Glucose and Diabetic Type 2 Diuretic Deficiencies in Children with PCOS in Children with Down Syndrome. Iran J Diabetes Metab. 2004;3(2):141-148.

27. Morin-Papunen LC, Vauhkonen I, Koivunen RM, et al. Insulin sensitivity, insulin secretion, and metabolic and hormonal parameters in healthy women and women with polycystic ovarian syndrome. Hum Reprod. 2000;15(6):1266-1274.

28. Velazquez E, Bellabarba GA, Mendoza S, et al. Postprandial triglyceride response in patients with polycystic ovary syndrome: relationship with waist-to-hip ratio and insulin. Fertil Steril. 2000;74(6):1159-1163.

29. Nati Datt, Ahmadian Das, Ahmadi Damah. Measured surface of insulin and mitochondrial risk factors in patients with PTSD. Scientific Journal of Science. 2006;8(1):11-6.

30. Alberti KGMM, Zimmet PZ. Definition, diagnosis and classification of diabetes mellitus and its complications. Part 1: diagnosis and classification of diabetes mellitus. Diabet Med. 1998;15(7):539-553. 